\title{
La mediación familiar: una intervención para abordar la ruptura de pareja
}

\author{
B. GonzÁlez Martín \\ Mediadora familiar. Abogada. Servicio de M ediación Familiar. \\ Unión de Asociados Familiares (UNAF). Madrid
}

\section{RESUMEN}

La ruptura de la pareja es una de las causas fre cuentes de estrés emocional y provoca, en numero sas ocasiones, sufrimiento psicológico y síntomas físicos asociados a ese malestar.

Los profesionales de los ámbitos jurídicos, psico lógicos y sociales, fundamentalmente, se vienen en frentando desde hace décadas en el ámbito de sus respectivos trabajos al fenómeno del divorcio y fru to del análisis de su experiencia, surge la reflexión sobre la necesidad de amortiguar tanto los costes emocionales como económicos del divorcio y evitar el daño que las rupturas difíciles y traumáticas ocasionan a los menores.

La mediación familiar aparece así como una nue va alternativa para gestionar los conflictos deriva dos de la ruptura; consiste en un modelo de inter vención estructurado que tiene como objeto ayudar a las parejas en situaciones de separación o divor cio a elaborar un acuerdo duradero y mutuamente aceptable para la nueva reorganización familiar, te niendo en cuenta las necesidades de cada uno de los miembros de la familia y, en especial, la de los hijos.

Se desarrolla a lo largo de varias entrevistas en las que están presentes los dos miembros de la pareja y el mediador, guiando éste la negociación que abarca a todos los temas que afectan a la ruptura. La mediación familiar otorga protagonismo a la pareja, favorece la adopción de acuerdos y tiene como presupuesto la consideración de que las par tes en situación de divorcio no son adversario, si no progenitores con un interés común: el de sus hijos.

Palabras clave: Divorcio. Mediación familiar. Intervención breve. Acuerdos. Hijos.

\section{Family mediation: an intervention to solve the couple breakup}

\begin{abstract}
Couple breakup is one the most common causes of emotional stress and, in many cases, it results in psychological suffering and physical signs associated to such disorder.

Since several decades ago, professionals in mainly the legal, psychological and social settings are being confronted in their respective jobs to the phenomenon of divorce. The analysis of their experience have driven to the reflection on the need both to alleviate the emotional costs of divorce and to avoid the damage that difficult and traumatic breakups cause on the minor.

Family mediation appears thus as a new alter native for managing conflicts derived from break ups; it includes a model of structured intervention aimed to help the couples in situations of separa tion or divorce to come to a lasting and mutually acceptable agreement for the new family reorgani zation, taking into account the needs of each of the family members and, in particular, the children.

It takes place through several interviews in which both members of the couple and the mediator are present, the latter guiding the negotiation and covering all the issues involved in the breakup. Family mediation allows the couple to play the lead, facilitates agreements and assumes that both parties in a divorce are not adversaries, but proge nitors with a common interest: their children.
\end{abstract}

Key words: Divorce. Family mediation. Short intervention. Agreements. Children. 


\section{INTRODUCCIÓN}

Como es bien sabido y así se ha expresado en algún artículo publicado en esta misma revista, "los trastornos mentales y el sufrimiento psicosocial están presentes de forma importante en las consultas de Atención Primaria, donde los médicos realizan intervenciones psicoterapéuticas más frecuentes de lo que se asume"'.

Pues bien, como sin duda podrían ratificar tanto estos facultativos como otros especialistas, una de las frecuentes causas de estrés emocional viene provocada por la ruptura matrimonial o cese de la convivencia, experiencia que es calificada por Rojas Marcos en su libro La pareja rota como una de "las más traumáticas, amargas y penosas que pueden sufrir los seres humanos". Este conocido psiquiatra describe muy gráficamente que "a continuación de la ruptura, tanto el hombre como la mujer atraviesan un periodo durante el cual se sienten terriblemente angustiados. Se quejan de no poder dormir, de haber perdido el apetito, de ser incapaces de concentrarse en el trabajo, o incluso de llorar a menudo sin razón aparente. En algunos casos, recurren al alcohol, a las drogas o a los tranquilizantes para apaciguar el dolor y ausentarse emocionalmente"2. Añade que también los hijos de las parejas en situación de ruptura pueden experimentar en los primeros momentos problemas de diversa índole tales como introversión, o falta de apetito o regresión o disminución en el rendimiento escolar, síntomas que normalmente son pasajeros pero que provocan en los padres miedo y ansiedad. Y concluye que "numerosos estudios han demostrado que las parejas rotas tienen una mayor predisposición a sufrir enfermedades físicas y mentales: hipertensión, úlcera de estómago, colitis, ataques de corazón, ansiedad y, sobre todo, depresión"’.

Para prevenir o, al menos, tratar de aliviar esas tensiones que provoca la ruptura de pareja existe un modelo de intervención profesional denominado mediación familiar, cuyo conocimiento puede resultar muy útil a los médicos de familia para su divulgación o derivación de aquellos pacientes que se encuentren en esa situación de separación o ruptura de pareja.

Como más adelante desarrollaremos, la mediación familiar puede considerarse un instrumento que faculta a las parejas en situación de separación llegar a un acuerdo sobre los efectos de la ruptura y, al mismo tiempo, facilita una comunicación más fluida que les permite mantener una relación posterior estable y pacífica para ejercer conjuntamente sus responsabilidades parentales. Además, la mediación provoca algún efecto terapéutico, pues ayuda a avanzar en el proceso de elaboración del duelo producido por la separación, ya que "se alienta a los participantes a reconocer los sentimientos negativos que acompañan a la ruptura"3.

\section{CONTEXTO HISTÓRICO}

La mediación familiar en su forma actual aparece en los Estados Unidos a finales de los años 60, país pionero junto con Canadá en la práctica de la mediación. A Europa llega a finales de los años 70 y, concretamente, en 1973 se abren en Gran Bretaña los primeros servicios de mediación familiar. A partir de esa fecha se va implantando en diversos países europeos y actualmente puede considerarse arraigada no sólo en Gran Bretaña, sino también en Francia, Alemania, Países Bajos y Noruega.

Por otra parte, el Consejo de Europa adoptó el día 21 de enero de 1998 la Recomendación $n^{\circ} \mathrm{R}$ (98) I sobre Mediación Familiar, que está suponiendo un gran impulso de esta intervención, pues establece entre otras, las siguientes directivas:

"11.i.-Instituir o promover la mediación familiar $o$, en su caso, reforzar la mediación familiar ya existente.

11.ii.- Adoptar o reforzar aquellas medidas que juzguen necesarias para la promoción y utilización de la mediación familiar como medio adecuado de resolución de litigios familiares."'.

La citada Recomendación parte de la idea de que el número de separaciones y divorcios en los Estados miembros sigue incrementándose, reconoce que los conflictos familiares tienen consecuencias importantes no sólo para las familias, sino también costes sociales y económicos para los Estados, considera que debe garantizarse la protección y bienestar de los menores y concluye que el recurso de la mediación familiar "puede en su caso: a) mejorar la comunicación entre los miembros de la familia; b) reducir los conflictos entre las partes en litigio; c) dar lugar a acuerdos amigables; d) garantizar el mantenimiento de relaciones personales entre los padres y los hijos; y e) reducir los costes económicos y sociales de la separación y del divorcio para las partes y los Estados y reducir el tiempo necesario para reglamentar los conflictos".

Centrándonos en España, hay que mencionar que, concretamente en Cataluña, se ha promulgado hace escasos meses la Ley 1/2001, de 15 de marzo, de Mediación Familiar, que entrará en vigor el próximo mes de diciembre y, además, se encuentran en la actualidad en fase de tramitación parlamentaria proyectos de leyes de mediación familiar en las Comunidades Autónomas del País Valenciano, Galicia y Navarra.

La Ley catalana de mediación familiar señala en su Exposición de Motivos que los equipos psicosociales, integrados por psicólogos y trabajadores so- 
ciales, adscritos a los Juzgados de familia han puesto en práctica un modelo de mediación familiar, que surge de la experiencia acumulada durante años y se aplica con un resultado positivo; añade que la ley tiene como objetivo "institucionalizar, potenciar y extender a toda Cataluña la mediación familiar" de Cataluña, organismo que asume la responsabilidad de fomentar y organizar el servicio público de esa actividad, sin perjuicio de las iniciativas privadas que hayan surgido o surjan en el futuro.

Independientemente de esa incipiente regulación legal, la práctica de la mediación en España está extendida por toda la geografía, existiendo servicios públicos de mediación familiar en las Comunidades Autónomas de Madrid, Cataluña, Asturias, Galicia, País Valenciano, País Vasco, Cantabria, Aragón y Murcia y, además, en Cádiz, Sevilla, Gran Canaria y Mallorca. En Madrid iniciaron la mediación "dos servicios subvencionados por el Ministerio de Asuntos Sociales, siendo destacable el Servicio de Mediación Familiar de la Unión de Asociaciones Familiares -UNAF- que actúa desde el año 1990 mediante un convenio-programa suscrito entre el Ministerio de Asuntos Sociales y la UNAF".

\section{DEFINICIÓN Y ESTRUCTURA DE LA MEDIACIÓN FAMILIAR}

La mediación familiar puede definirse como aquel proceso voluntario, extrajudicial y confidencial del que se pueden valer aquellas parejas, casadas o no, que han decidido separarse o que están ya separadas o divorciadas, para negociar y decidir por sí mismas y con la ayuda de un profesional neutral, imparcial y cualificado, todos los efectos de la ruptura de pareja.

La mediación es un proceso estructurado con una metodología muy precisa que se desarrolla a lo largo de nueve entrevistas, por término medio. Las primeras entrevistas se centran en explicar con claridad tanto los objetivos de la mediación como el papel del mediador y se evalúa la actitud de la pareja frente a la mediación y si cumplen los requisitos exigidos para la intervención. En esas entrevistas el mediador verifica si la decisión de la separación es una decisión firme y querida o, al menos, aceptada, por ambos miembros de la pareja, y se exploran otras alternativas a la crisis convivencial, si las hubiera.

Una vez descartadas otras posibles soluciones, comienza la mediación propiamente dicha; para ello la pareja ha de firmar un documento en el que expresan su consentimiento para realizar la intervención y aceptan las reglas básicas de ésta cuyo incumplimiento supone la suspensión de la media- ción. Entre esas reglas se encuentran las siguientes: - La voluntariedad, pues se parte de la idea de que la intervención será eficaz si ambos miembros de la pareja desean comenzar, continuar y finalizar la mediación. La voluntariedad da protagonismo a la pareja y también responsabilidad.

- La confidencialidad, regla ésta que concierne especialmente al mediador, quien se compromete a no declarar como testigo o perito ante un tribunal si la mediación se suspendiera, permitiendo por tanto a la pareja tratar con libertad todos los temas de negociación.

- La cooperación en el tratamiento con transparencia de los diversos puntos del acuerdo, incluidos los económicos, comprometiéndose la pareja a aportar información y documentación útil al respecto.

—La obligación de suspender acciones judiciales mientras dura el proceso de mediación.

-El compromiso de que las sesiones transcurran en un clima de respeto mutuo.

Firmado el consentimiento para la mediación, la pareja con asistencia del mediador empieza a negociar todos aquellos aspectos afectados por la ruptura como son: el ejercicio de la patria potestad o, dicho en otros términos, la forma de abordar las responsabilidades parentales; es decir, todo lo que tiene que ver con las cuestiones fundamentales de la vida de los hijos: la salud, la educación, las relaciones con la familia extensa; la guarda y custodia de los hijos o la decisión sobre con cuál de los dos progenitores van a vivir los menores habitualmente; el tiempo y modo en que aquéllos se van a relacionar con el otro progenitor con el que no conviven habitualmente o, como se denomina jurídicamente, el régimen de visitas, estancias y comunicaciones; el uso de la vivienda familiar; las necesidades económicas de los hijos y la contribución a éstas, o dicho en términos jurídicos, la pensión alimenticia. Además se tratan otros aspectos económicos, como la pensión compensatoria, que es la que corresponde al cónyuge al que la separación o divorcio le ocasione un grave desequilibrio económico, o la distribución patrimonial o el reparto de los bienes comunes.

Todos esos temas se hablan en profundidad en mediación familiar, requiriéndose la presencia de ambos miembros de la pareja en tantas entrevistas como sean precisas. La finalización de la mediación supone la redacción por el mediador de un documento denominado proyecto de entendimiento parental en el que se plasman todos los acuerdos alcanzados por la pareja, documento que debe presentarse en el Juzgado para que el juez lo apruebe y le otorgue validez.

Es conveniente precisar que la mediación no es una terapia, pues aquella es una intervención cen- 
trada en el presente y en el futuro más que en el pasado, y no tiene como objetivo el cambio cognitivo, conductual y afectivo ni la introspección de patrones de conducta ${ }^{3}$, pero como hemos señalado en la introducción, tiene efectos terapéuticos porque al alentar la participación de los dos miembros de la pareja en la adopción de los acuerdos, permite restablecer la comunicación entre ambos, estando, por otra parte, atento el mediador a las respuestas emocionales que estas entrevistas provocan.

\section{SUPOSICIONES BÁSICAS Y LA FUNCIÓN DEL MEDIADOR}

La mediación familiar descansa sobre una serie de presupuestos básicos que podríamos resumir como sigue:

-La separación o el divorcio no han de suponer para los hijos la pérdida de alguno de sus progenitores, debiendo conservarse intactos los vínculos entre aquéllos y éstos.

- Las parejas que se separan pueden encontrar por sí mismas las bases del acuerdo que ha de regir sus relaciones posteriores.

- Los procesos judiciales contenciosos enfrentan a las partes y provocan una mayor conflictividad, que no favorece el entendimiento y la comunicación entre los padres separados y divorciados respecto de sus hijos.

Esas ideas centrales son las que el mediador transmite a la pareja que acude a mediación, debiendo reunir aquél, por tanto, una serie de características personales y profesionales, pues en buena medida el éxito de una mediación depende de él, al ser quien guía la negociación.

En primer lugar, el mediador ha de tener una formación específica independientemente de su formación de origen, y así generalmente se exige que tal profesional sea licenciado o diplomado en ciencias humanas, sociales o jurídicas y, además, reciba un curso o master de especialización que incluya, entre otros extremos, conocimientos básicos de derecho de familia, teoría de la negociación, técnicas de entrevista y gestión de conflictos.

En relación con la formación del mediador, la Recomendación del Consejo de Europa ya citada dispone que: "Debe haber mecanismos adecuados que garanticen la existencia de:

-Procedimientos para la selección, la forma ción y la calificación de los mediadores.

- Normas de 'práctica adecuada' deberían ser elaboradas y cumplidas por los mediadores. "“.

Por su parte, la Ley de Mediación Familiar de Cataluña señala en su artículo 7:
"1. El mediador o mediadora debe ser una perso na que ejerza de abogado, de psicólogo, de trabaja dor social, de educador social o de pedagogo y que esté colegiada en el colegio profesional respectivo.

2. La condición de mediador o mediadora ha de ser declarada de acuerdo con la experiencia profe sional y la formación específica que se establezcan por reglamento" ${ }_{5}$.

Por otra parte, el mediador ha de gozar de unas determinadas cualidades personales, como son: empatía, es decir, capacidad afectiva y emotiva; y facilidad para la comunicación y para poder estimularla entre la pareja. Algún autor menciona también la utilización de un lenguaje neutro, la escucha activa, el control de las emociones propias y del proceso y la capacidad para detectar los bloqueos de la comunicación ${ }^{7}$.

Finalmente, el mediador no tiene poder de decisión -siendo ésta la diferencia que separa a la mediación del arbitraje-y debe ser neutral e imparcial en su interacción con la pareja, lo que significa que no debe dejarse influir ni tampoco trasladar a la mediación sus valores, sus emociones o sus prejuicios y, al mismo tiempo, no puede tomar partido por ninguno de los dos miembros de la pareja, debiendo equilibrar el tiempo en el uso de la palabra de cada uno de ellos.

\section{INDICACIONES Y CONTRAINDICACIONES DE LA MEDIACIÓN FAMILIAR}

La filosofía que rige la Mediación resulta tan atrayente que puede dar lugar a una visión excesivamente optimista de la misma, llegando al error de creer que la mediación es la alternativa por excelencia para la resolución de todos los problemas o conflictos familiares ${ }^{8}$.

Según Lisa Parkinson, los enfrentamientos que acompañan la ruptura convivencial tienen raíces muy profundas, existen conflictos estructurados en los que ambos cónyuges tienen una profunda necesidad emocional de seguir peleando, porque su vida carecería de significado si uno de ellos renunciase a la lucha, "en estos casos, incluso los mediadores más expertos, pueden fracasar al intentar resolver conflictos enmarañados a los que ninguna de las partes quieren poner fin".

Es por ello, por lo que debemos reconocer que la mediación familiar -como cualquier especialidadtiene sus limitaciones; esencialmente, porque trabaja partiendo de la libre voluntad de las partes, y allí donde ésta esté mediatizada por alguna circunstancia, la mediación estará contraindicada.

En este sentido, la mediación familiar no parece la solución más aconsejable para aquellos casos en los que: 
-Alguno de los miembros de la pareja no ejerza control sobre su voluntad y, por lo tanto, sean incapaces de asumir compromisos adquiridos o, incluso, de adquirir compromiso alguno. Es por esto, por lo que no debe de iniciarse una mediación si se tiene la certeza de que uno o ambos miembros de la pareja, padecen comportamientos disfuncionales tales como alcoholismo, toxicomanías, etc.

- Tampoco es aconsejable la mediación en todos aquellos supuestos, en los que uno de los miembros de la pareja, o sus hijos, sean víctimas de violencia familiar, porque las decisiones estarán inevitablemente condicionadas por el desequilibrio de poder que existe entre la pareja, llegando a influir en los acuerdos el temor al otro, con el consiguiente riesgo para los miembros de la unidad familiar, víctimas de la violencia, y el incremento de la probabilidad de incumplimiento de los acuerdos.

En esos casos hay que derivar a los clientes a otro tipo de servicios, como son: terapia de pareja o familiar, si no desean la separación y quieren mejorar su relación o terapias específicas si padecen alguna dificultad emocional o enfermedad.

\section{CONCLUSIONES}

La práctica de la mediación en los países en los que está implantada ha demostrado ser un instrumento muy útil para gestionar y abordar la ruptura de pareja. Por un lado, los acuerdos alcanzados, al ser decididos y negociados por los propios protagonistas, son más satisfactorios y se cumplen con más frecuencia. Por otro, ello redunda en beneficio de los progenitores y, en especial, de los menores quienes perciben que aquéllos siguen actuando como padres.

Al respecto, conviene insistir en que, como aparece estadísticamente comprobado, "las reacciones de los hijos ante la separación de sus padres están directamente relacionadas con la intensidad y calidad con que venga revertido el procedimiento de separación o divorcio. No son la separación o el divorcio en sí mismos, sino la mala manera de ejecutarlos y la errónea actuación posterior de los padres lo que conlleva convivencias negativas para los menores. Los hijos pueden superarlo si los padres cooperan. Si la separación es conflictiva, la menor y el menor la experimentan en términos de pérdida: se rompen los vínculos afectivos con el padre o la madre, se pierde un modelo de identificación, le crea inseguridades, pérdida, etc." ${ }^{10}$.

Por ello, resulta imprescindible divulgar tanto la mediación familiar como los fines que persigue: la importancia de fomentar el ejercicio conjunto de las responsabilidades parentales tras la ruptura y la necesidad de que los hijos conserven a ambos padres después de la separación.

\section{CORRESPONDENCIA:}

Begoña González Martín

Mediadora Familiar. Abogada

Servicio de Mediación Familiar (UNAF)

C/ Alberto Aguilera, 3, $1^{\circ}$ izda.

28015 Madrid

E-mail: bgonzalezm@icam.es

\section{Bibliografía}

1. Aiarzaguena Sarriugarte JM, Ariño Viar J. D-E-P-E-N-A-S, hormonas y emociones: una guía práctica para el abordaje del malestar psicológico en Atención Primaria. MEDIFAM 1999; 9: 68-74.

2. Rojas Marcos L. La pareja rota. Familia, crisis y superación. $4^{a}$ Ed. Madrid: Colección Espasa Hoy, Espasa Calpe, 1994.

3. Folberg J, Taylor A. Mediación. Resolución de Conflictos sin litigio. $1^{a}$ Ed. México D.F: Editorial Limusa, Grupo Noriega Editores, 1992

4. Recomendación $n^{\circ} \mathrm{R}(98)$ I sobre Mediación Familiar. Textos adoptados por el Comité de Ministros del Consejo de Europa. Publishing Council of Europe. Estrasburgo: Consejo de Europa, 1998

5. Ley $1 / 2001$, de 15 de marzo, de Mediación Familiar de Cataluña. BOE 91 de 16 de abril de 2001; 13797-802.

6. Villagrasa Alcaide C, Vall Rius $\mathrm{AM}^{\mathrm{a}}$. La mediación familiar: Una nueva vía para gestionar conflictos familiares. La Ley. Revista jurídica española de doctrina, jurisprudencia y bibliografía. (Madrid) 2000; número 5049: 1-7.
7. Redorta Lorente J. Mediación: algunos conceptos básicos orientados a la práctica. Ponencia. I Congreso de Mediación Familiar. 1999 abril 22-24; Valencia; Asociación de Operadores Familiares y Sociales, 1999.

8. Abril Pérez del Campo C. Mediación familiar: un recurso para la resolución de los conflictos familiares. Ponencia. Curso sobre intervención de los técnicos en los procedimientos de derecho de familia: evaluación pericial frente a la mediación. 1994 noviembre 28-30; Madrid; Seminario de Formación del Centro de Estudios Judiciales, 1994.

9. Parkinson L. Mediating with high conflict couples. Ponencia. Congreso Internacional de Mediación Familiar. 1999 octubre 6-8; Barcelona; Centro de Estudios Jurídicos y Formación Especializada del Departamento de Justicia de la Generalitat de Cataluña, 1999.

10. Espada Giner S. La mediación familiar. Aequalitas. Revista jurídica de igualdad de oportunidades entre mujeres y hombres. (Zaragoza) 2000; número 5; 41-8. 